\title{
Stochastic Model for Cancer Cell Growth through Single Forward Mutation
}

Jayabharathiraj Jayabalan

Pondicherry University, jayabharathi8@gmail.com

Follow this and additional works at: http://digitalcommons.wayne.edu/jmasm

Part of the Applied Statistics Commons, Social and Behavioral Sciences Commons, and the Statistical Theory Commons

\section{Recommended Citation}

Jayabalan, J. (2017). Stochastic model for cancer cell growth through single forward mutation. Journal of Modern Applied Statistical Methods, 16(1), 578-588. doi: 10.22237/jmasm/1493598660

This Emerging Scholar is brought to you for free and open access by the Open Access Journals at DigitalCommons@WayneState. It has been accepted for inclusion in Journal of Modern Applied Statistical Methods by an authorized editor of DigitalCommons@WayneState. 


\title{
Stochastic Model for Cancer Cell Growth through Single Forward Mutation
}

\author{
Jayabharathiraj Jayabalan \\ Pondicherry University \\ Puducherry, India
}

\begin{abstract}
A stochastic model for cancer cell growth in any organ is presented, based on a single forward mutation. Cell growth is explained in a one-dimensional stochastic model, and statistical measures for the variable representing the number of malignant cells are derived. A numerical study is conducted to observe the behavior of the model.
\end{abstract}

Keywords: Mutation, probability generating function, differential-difference equation

\section{Introduction}

Cancer has complex and stochastic cell growth mechanisms. Malignant cancer cells arise from several mutations in the gene of a cell. It has been shown that a normal cell requires more than one stage to become a malignant cell (Tan \& Brown, 1987). Stochastic growth is observed in malignant cells, and deterministic exponential growth is observed in normal cells. Most developed models are mixed, representing both deterministic and stochastic cell growth. The type of growth in a normal cell population depends on whether mutation has taken place.

Let $[X(t), \mathrm{t}>0]$ be a stochastic process denoting the number of normal cells in an organ at time $t$, and $[Y(t), \mathrm{t}>0]$ be a another stochastic process denoting the number of malignant cells in an organ at time $t$. Let us define a bivariate cell growth process $\{(X(t), Y(t)), t>0\}$ representing the number of normal and malignant cells at time $t$. The growth of cells can be studied using the birth-anddeath process. In literature, the process of malignant cell growth has been studied with homogeneous and non-homogeneous birth, death and mutation processes, but it seems most applicable when the study is conducted under a time-dependent

Jayabharathiraj Jayabalan is a Research Scholar in the Department of Statistics. Email at jayabharathi8@gmail.com. 


\section{JAYABHARATHIRAJ JAYABALAN}

environment. The birth-and-death process has been used to study the stochastic growth of a population, and the average and variance of the size of a population has been obtained for a given time period (Kendall, 1949). A similar approach can be applied to obtain the average and variance of the number of malignant cells in an organ at time $t$.

Assume there are $x_{0}$ number of normal cells and $y_{0}$ number of malignant cells in any organ at time $t=0$ (initially). The model representing the cell division process for normal and malignant cells can be explained using either one or two variables. Consider a single forward mutation process for the transformation of normal cells into malignant cells, which reflects in the growth of the cell population. If a malignant cell is formed from a normal cell, and it remains in the same state till extinction, then there is not backward process of mutation. Let us assume that the normal cell has deterministic exponential growth; the expected number of cells in an organ at time $t$ is then defined by (Serio, 1984),

$$
\hat{X}(t)=x_{0} \exp \left\{\int_{0}^{t}\left[\left(b_{N}() d_{N}()\right)+t_{N M}()\right] d\right\}
$$

If it is assumed the malignant cells also show deterministic growth, i.e., the cell growth at the malignant stage is deterministic and exponential, then the expected number of malignant cells is as follows,

$$
\hat{Y}(t)=y_{0} \exp \left\{\int_{0}^{t}\left(b_{M}() d_{M}()\right) d\right\}
$$

\section{Assumptions}

The model is developed based on the following assumptions:

1. Let the growth rate of normal cells from normal cells be $b_{N}(t)$, and the probability of growth of normal cells from normal cells in $d t$ be $b_{N}(t) d t+o(d t)$. Let the death rate of normal cells be $d_{N}(t)$ and probability of the death of normal cells in $d t$ be $d_{N}(t) d t+o(d t)$.

2. Let the growth rate of malignant cells from the malignant cells be $b_{M}(t)$, and probability of growth of malignant cells from malignant cells be $b_{M}(t) d t+o(d t)$. Let the death rate of malignant cells be $d_{M}(t)$ 


\section{STOCHASTIC MODEL FOR CANCER CELL GROWTH}

and probability of the death of malignant cells in $d t$ be $d_{M}(t) d t+o(d t)$.

3. Let the growth rate of the normal cell population be represented by $\left\{\left(b_{N}(t)-d_{N}(t)\right)+\mu t_{N M}(t)\right\}$, and the growth rate of the malignant cell population by $\left(b_{M}(t)-d_{M}(t)\right)$.

4. In a very small interval $(t+d t)$, let the probability of a mutation which transforms a normal cell into a malignant cell be $x \mu t_{N M}(t) d t+o(d t)$, where $X(t)=x_{0}$ at $t=0$.

When a mutation takes place in a normal cell population, the number of normal cells is decreased by one and the number of malignant cells is increased by one. Assume that the growth rate for normal and malignant cell populations are different. For any organ, a certain number of cells is required for normal, proper functioning; normal functioning of any organ depends upon the number of cells.

The expected population size at time $t$ can be described as $X(t)+Y(t)$. Assuming a deterministic growth for normal and malignant cells, then

$$
\begin{aligned}
T_{c} & =\hat{X}(t)+\hat{Y}(t) \\
& =x_{0} \exp \left\{\int_{0}^{t}\left[\left(b_{N}() d_{N}()\right)+t_{N M}()\right] d\right\} \\
& +y_{0} \exp \left\{\int_{0}^{t}\left(b_{M}() d_{M}()\right) d\right\}
\end{aligned}
$$

where $X(t)=x_{0}$ and $Y(t)=y_{0}$ at $t=0$. Hence, the number of normal cells in the population of an organ at time $t$ is as follows

$$
\begin{aligned}
\hat{X}(t) & =x_{0} \exp \left\{\int_{0}^{t}\left[\left(b_{N}() d_{N}()\right)+t_{N M}()\right] d\right\} \\
& +y_{0} \exp \left\{\int_{0}^{t}\left(b_{M}() d_{M}()\right) d\right\} \hat{Y}(t)
\end{aligned}
$$

Assuming the above relation holds, there exists a stochastic dependence between $X(t)$ and $Y(t)$. There is no need to observe the variables $X(t)$ and $Y(t)$ as a 


\section{JAYABHARATHIRAJ JAYABALAN}

two-dimensional stochastic process; it is enough to consider one-dimensional stochastic process for the malignant cell population $[Y(t), \mathrm{t}>0]$ with the above relation. The above discussions deal with a non-homogeneous environment, and look more complex in mathematical derivations. For simplicity, let us assume a homogeneous environment with respect to birth, death and mutation parameters.

\section{Stochastic Model}

Let $f_{M}(y, t)=P\{Y(t)=y\}$ denote the probability density function of $Y(t)$. Assume that $f_{M}(y, t)$ exists and is differentiable with respect to both $y$ and $t$; from Assumption 4, obtain the following relation (Armitage, 1952):

$$
\begin{aligned}
& P[y<Y(t+d t)<y+d y]=f(y, t+d t) d y
\end{aligned}
$$

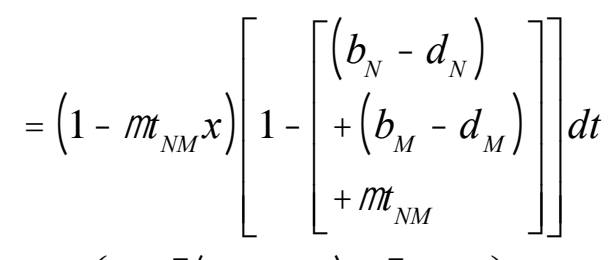



$$
\begin{aligned}
& +t_{N M}(x+1) f_{M}(y \quad 1, t) d y d t+((O d t)) d y+o(d y)
\end{aligned}
$$

By passing the limit on both sides in above equation, we obtain the differential-difference equation in the form as follows

$$
\begin{aligned}
& \frac{\partial f_{M}(y, t)}{\partial t}+\left[\begin{array}{ll}
\left(b_{N}\right. & d_{N}
\end{array}\right)+\left(\begin{array}{ll}
b_{M} & \left.d_{M}\right)+t_{N M}
\end{array}\right] y \frac{\partial f_{M}(y, t)}{\partial t} \\
& =(x+1) t_{N M} f_{M}\left(\begin{array}{ll}
y & 1, t)
\end{array}\right)\left[\begin{array}{ll}
\left(\begin{array}{ll}
b_{N} & d_{N}
\end{array}\right) \\
+\left(\begin{array}{ll}
b_{M} & d_{M}
\end{array}\right) \\
+ & t_{N M} \\
+ & t_{N M} x
\end{array}\right] f_{M}(y, t)
\end{aligned}
$$

By using the relations given in equation (1), the above equation becomes, 


$$
\begin{aligned}
& \frac{\partial f_{M}(y, t)}{\partial t}+\left[\begin{array}{ll}
\left(b_{N}\right. & \left.d_{N}\right) \\
+\left(\begin{array}{ll}
b_{M} & d_{M}
\end{array}\right) \\
+t_{N M}
\end{array}\right] y \frac{\partial f_{M}(y, t)}{\partial y} \\
& =t_{N M}\left[\begin{array}{c}
x_{0} \exp \left\{\left(\begin{array}{ll}
b_{N} & \left.\left.d_{N}\right) t\right\} \\
+y_{0} \exp \left\{\left[\begin{array}{ll}
\left(b_{M}\right. & d_{M}
\end{array}\right)\right] t \\
+ & t_{N M}
\end{array}\right]\right\} \\
y+1
\end{array}\right] f_{M}\left(\begin{array}{ll}
y & 1, t)
\end{array}\right]
\end{aligned}
$$

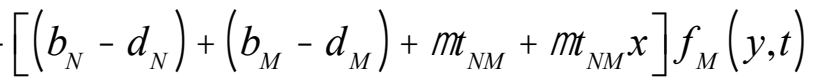

$$
\begin{aligned}
& \frac{\partial f_{M}(y, t)}{\partial t}+\left[\begin{array}{ll}
\left(b_{N}\right. & \left.d_{N}\right) \\
+\left(\begin{array}{ll}
b_{M} & d_{M}
\end{array}\right) \\
+t_{N M}
\end{array}\right] y \frac{\partial f_{M}(y, t)}{\partial y}
\end{aligned}
$$

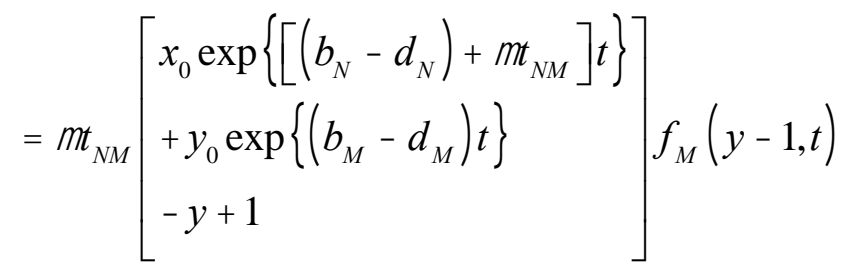

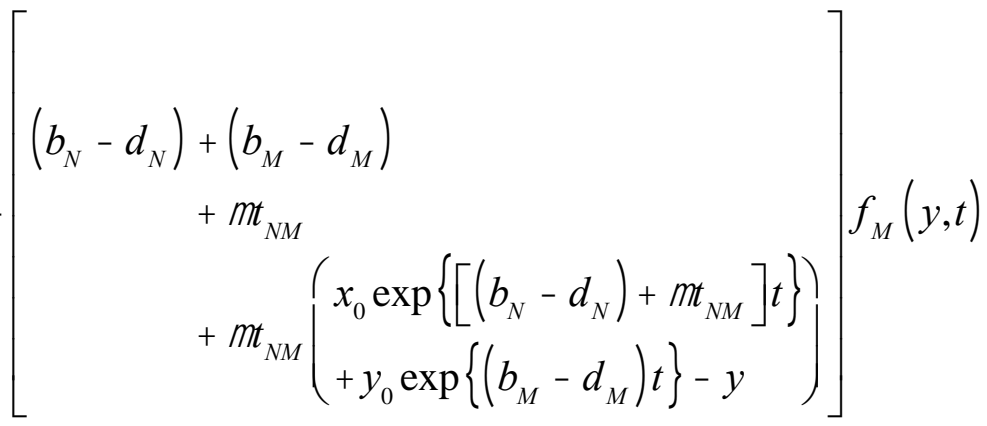

The boundary condition for the above equations is

$$
\begin{aligned}
& f_{M}(y, t)=0, \text { for } y<0, t \geq 0 \\
& \lim _{y \rightarrow \infty} y^{i} f_{M}(y, t)=0, \text { for all } i \geq 0, t \geq 0 .
\end{aligned}
$$




\section{JAYABHARATHIRAJ JAYABALAN}

The interest is to obtain the statistical moments such as mean and variance of malignant cells for a given time $t$. The probability generating function is

$$
\phi(s, t)=\int_{-\infty}^{\infty} s^{y} f(y, t) d y: 0 \leq s \leq 1
$$

The partial derivative $\phi(s, t)$ with respect to $s$ exists and from the boundary condition,

$$
\int_{\infty}^{\infty} y s^{y} \frac{\partial f(y, t)}{\partial s} d y=\left[(s, t)+s \log s \frac{\partial(s, t)}{\partial s}\right] .
$$

Multiply both sides of equation (1) by $s^{y}$ and integrate, which yields the following differential equation for the generating function as

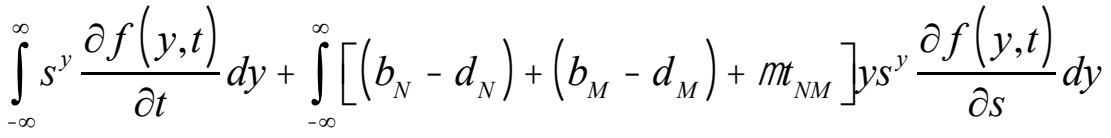



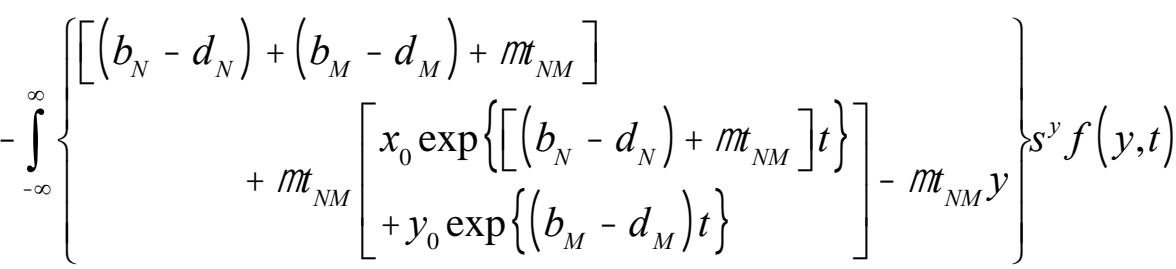




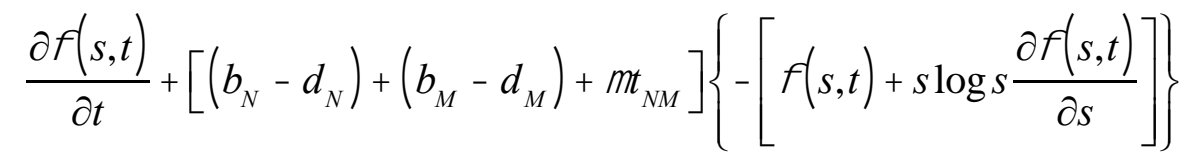

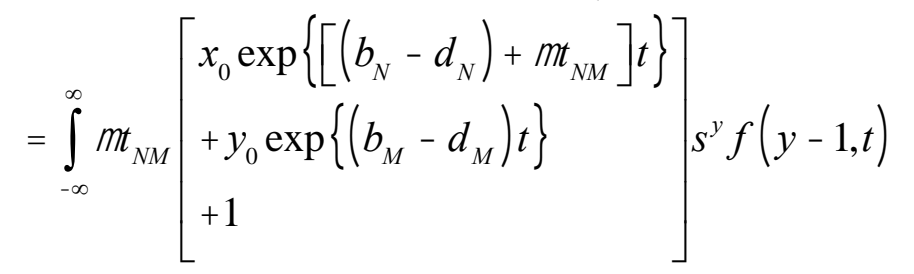

$$
\begin{aligned}
& \int_{\infty}^{\infty} t_{N M} y s^{y} f\left(\begin{array}{ll}
y & 1, t
\end{array}\right)
\end{aligned}
$$



$$
\begin{aligned}
& +\int_{\infty}^{\infty} t_{N M} y s^{y} f(y, t)
\end{aligned}
$$

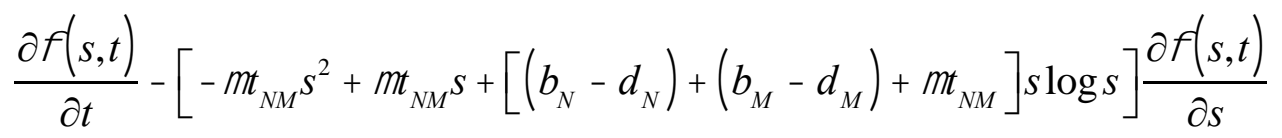



$$
\begin{aligned}
& \frac{\partial(s, t)}{\partial t}\left[t_{N M} s+\left[\begin{array}{ll}
b_{N} & \left.d_{N}\right)+\left(\begin{array}{ll}
b_{M} & \left.d_{M}\right)+t_{N M}
\end{array}\right] s \log s \quad t_{N M} s^{2}
\end{array}\right] \frac{\partial(s, t)}{\partial s}\right. \\
& =t_{N M}\left[x _ { 0 } \operatorname { e x p } \left\{\left[\left(\begin{array}{ll}
b_{N} & \left.d_{N}\right)+t_{N M}
\end{array}\right] t\right\}+y_{0} \exp \left\{\left(\begin{array}{ll}
b_{M} & \left.\left.\left.d_{M}\right) t\right\}\right]
\end{array}\right]\left(\begin{array}{ll}
s & 1
\end{array}\right)(s, t)\right.\right.\right.
\end{aligned}
$$

To obtain the moments, use the cumulant generating function of $y(t)$. Let $K(u, t)=\log \phi(s, t)$, where $s=e^{u}$. On simplification (Bharucha-Reid, 1960),

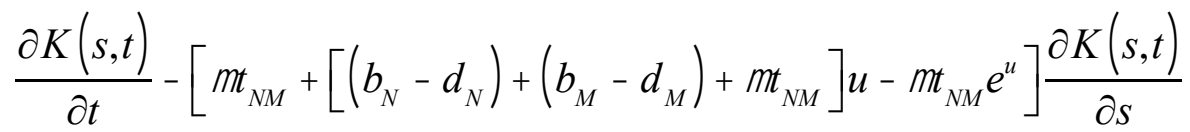

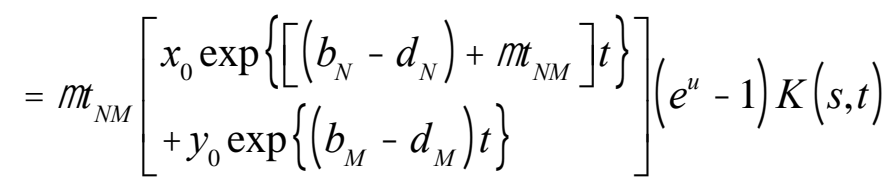




\section{JAYABHARATHIRAJ JAYABALAN}

\section{Statistical Moments}

The moments of the model can be obtained by expanding the cumulant generating function $K(u, t)$ on both sides of the expression as $K(u ; t)=u E(Y(t))+\frac{1}{2} u^{2} \operatorname{Var}(Y(t))+L$, comparing the coefficient of the power of $u$ 's and $v$ 's, and equating coefficients on both sides of the equation. In this way we arrive at the following linear differential equations of constant parameters

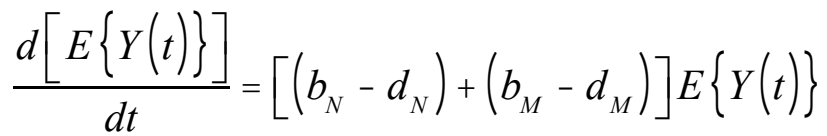

$$
\begin{aligned}
& +t_{N M}\left[\begin{array}{l}
x_{0} \exp \left\{\left[\begin{array}{ll}
\left(b_{N}\right. & \left.d_{N}\right)+t_{N M}
\end{array}\right] t\right\} \\
+y_{0} \exp \left\{\left(\begin{array}{ll}
b_{M} & d_{M}
\end{array}\right) t\right\}
\end{array}\right]
\end{aligned}
$$

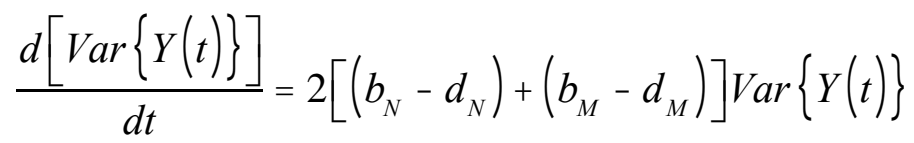

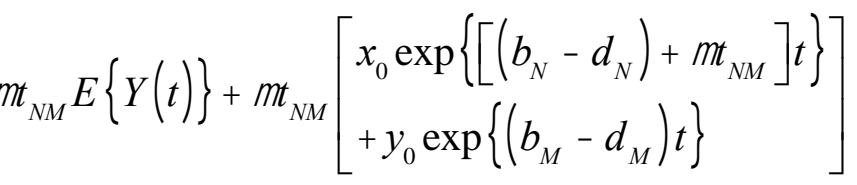

Solving the differential equation in (14) and (15) gives the average number of malignant cells and variance of number of malignant cells at a given time $t$. On solving above equation,

$$
\begin{aligned}
E[Y(t)] & =C_{1} \exp \left\{\left[\left(b_{N}-d_{N}\right)+\left(b_{M}-d_{M}\right)\right] t\right\} \\
& -\mu t_{N M}\left[\frac{x_{0} \exp \left\{\left[\left(b_{N}-d_{N}\right)+\mu t_{N M}\right] t\right\}}{\left[\left(b_{M}-d_{M}\right)-\mu t_{N M}\right]}+\frac{y_{0} \exp \left\{\left(b_{M}-d_{M}\right) t\right\}}{\left(b_{N}-d_{N}\right)}\right]
\end{aligned}
$$




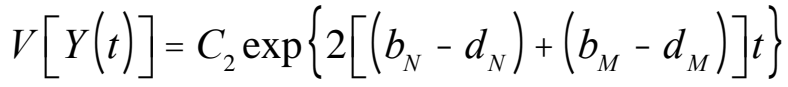

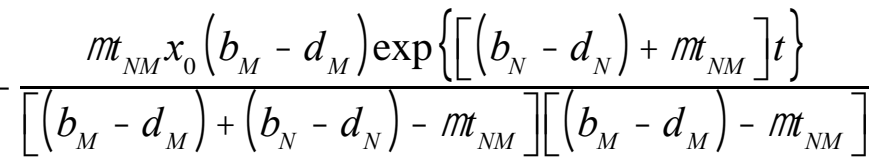

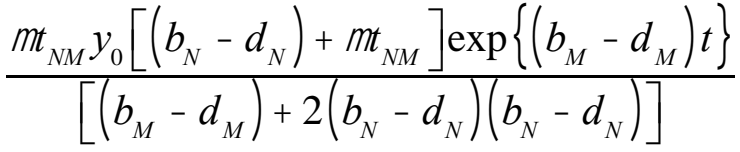

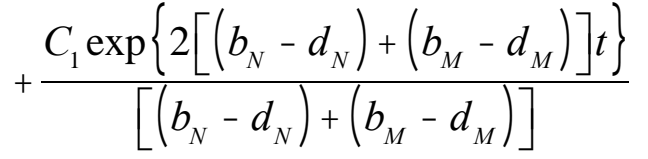

The integration constants $C_{1}$ and $C_{2}$ will be obtained using the boundary conditions of the differential equations.

\section{Numerical Study}

For the fixed parameters and changing time, the changes are observed in the average, and expected and variance numbers of malignant cells in any organ are presented. The numerical study was conducted using Mathematica 8.0 software for solving the differential equations as given above in equations (14) \& (15) numerically. The average and variance of number of malignant cells for fixed values of the parameters, $b_{N}=0.0001, d_{N}=0.0001, b_{M}=0.04, d_{M}=1.0 \times 10^{-7}$, $x_{0}=1.0 \times 10^{5}, y_{0}=1.0 \times 10^{5}$, and varying values of mutation rate and time are presented.

For the good maintenance of normal cell level, growth rate and death rate of normal cells are assumed to be equal, and large birth rate values for malignant cells and mutation rate are presented in the Figure 1. From the Figure, it is observed that there is a positive relationship between time and average number of malignant cells; a positive relationship between time and variance of number of malignant cells at lower values of mutation rates, and so on.

\section{Conclusion}

Birth, death, and single mutation processes with different growth rates are considered, to the study the growth of malignant cells by assuming $X(t)$ is dependent on $Y(t)$. The usual two dimensional models are replaced by a one dimensional model representing normal and malignant cells with interest in 


\section{JAYABHARATHIRAJ JAYABALAN}

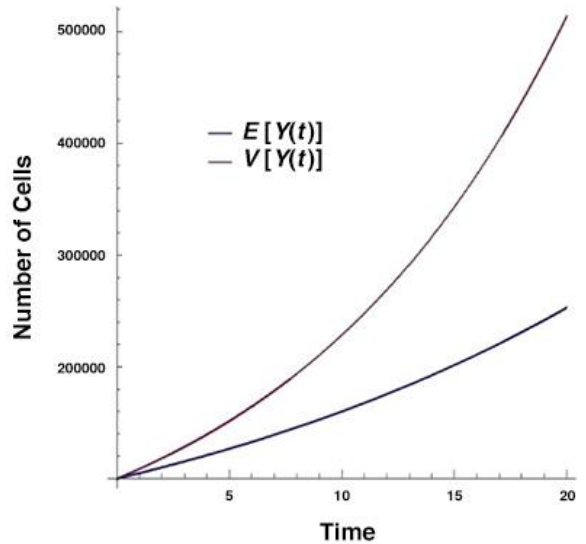

a) $\mu t_{N M}=0.0004$



c) $\mu t_{N M}=0.06$



e) $\mu t_{N M}=0.1$

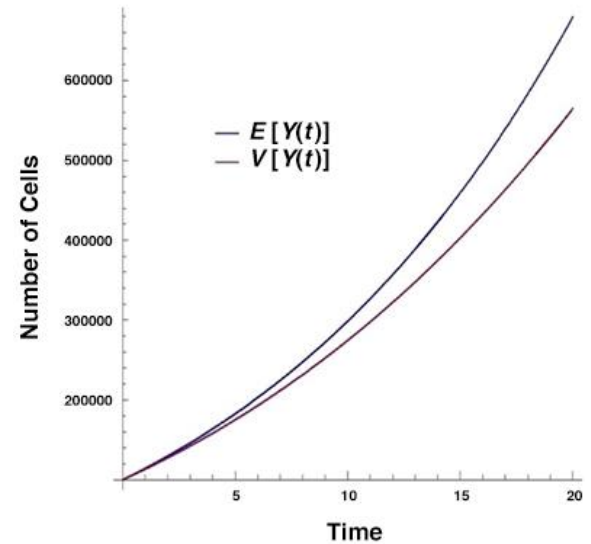

b) $\boldsymbol{\mu} \boldsymbol{t}_{N M}=\mathbf{0 . 0 4 9}$

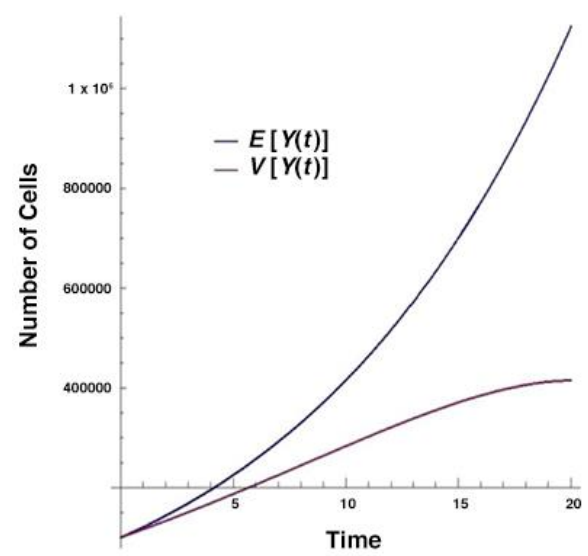

d) $\mu t_{N M}=0.08$

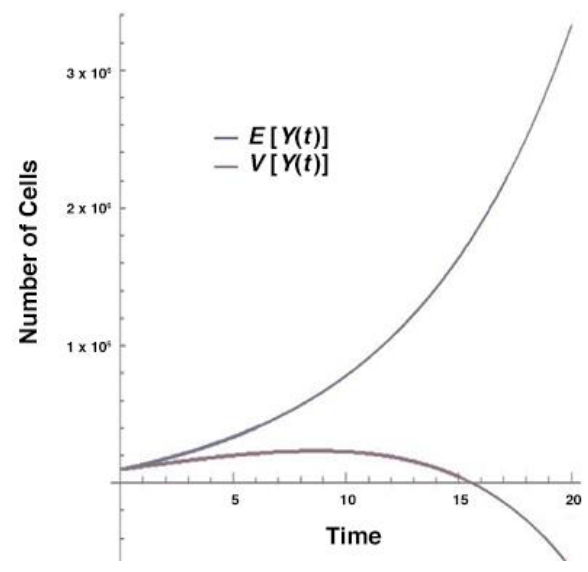

f) $\mu t_{N M}=0.15$

Figure 1. Variation in the moments with respect to time. 


\section{STOCHASTIC MODEL FOR CANCER CELL GROWTH}

malignant cell population. The statistical measure shows that volatility of the malignant population decreases as the mutation rate increases, and average number of malignant cells increases drastically as the mutation rate increases. The results of this study may help to understand the behavior of malignant cells over a period of time with various decision parameters.

\section{References}

Armitage, B. P. (1952). The statistical theory of bacterial populations subject to mutation. Journal of the Royal Statistical Society. Series B (Methodological), 14(1), 1-40.

Bharucha-Reid, A. T. (1960). Elements of the theory of markov processes and their applications. New York: McGraw-Hill.

Kendall, D. G. (1949). Stochastic processes and population growth. Journal of the Royal Statistical Society. Series B (Methodological), 11(2), 230-282.

Serio, G. (1984). Two-stage stochastic model for carcinogenesis with timedependent parameters. Statistics \& Probability Letters, 2(2), 95-103. doi: 10.1016/0167-7152(84)90057-9

Tan, W. Y. \& Brown, C. C. (1987). A non-homogeneous two-stage carcinogenesis model. Mathematical Modelling, 9(8), 631-642. doi:

10.1016/0270-0255(87)90463-5 